\title{
PHASE SEPARATION IN POLYMER SOLUTIONS. I. LIQUID-LIQUID PHASE SEPARATION OF PPO POLY (2, 6-DIMETHYL 1, 4-PHENYLENE OXIDE) IN BINARY MIXTURES WITH TOLUENE AND TERNARY MIXTURES WITH TOLUENE AND ETHYL ALCOHOL
}

\author{
P. T. VAN EMMERIK and C. A. SMOLDERS \\ Twente University of Technology, Enschede, Netherlands
}

\section{SYNOPSIS}

In the system poly (2, 6-dimethyl-1, 4-phenylene oxide) (PPO)-toluene three phase separation lines can be detected: the melting point curve, the cloud point curve, and the spinodial. Because crystallization of PPO occurs very slowly, a phase transition will always be initiated by liquid-liquid phase separation. Depending upon the experimental circumstances two mechanisms are possible, either nucleation and growth (extremely slowly in this system) or the spinodial decomposition mechanism. In the system PPO-toluene-ethanol, liquid-liquid phase separation occurs leading to two bulk liquid fractions. Only in mixtures containing very small concentrations of alcohol can three phase separation curves be detected from which the position of the cloud point curve of the system PPO-toluene can be confixmed.

\section{INTRODUCTION}

Depending upon the experimental circumstances liquid-liquid phase separation can be initiated by one of the following mechanisms: nucleation and growth [1], well known in a number of polymer solvent systems, or spinodial decomposition, which occurs in certain metal alloys and glasses and is described by the theory of Cahn [2]. In a few recent articles the onset of the phase separation of poly (2,6-dimethyl-1,4-phenylene oxide) (PPO)-caprolactam mixtures was described and explained by a spinodial decomposition mechanism $[3,4]$. An adaption of the theory of Cahn to solutions of high polymers has been given [5].

The present study was initiated to learn more about the thermodynamics of liquid-liquid phase separation of PPO-solvent mixtures. Some results concerning the phase equilibria of PPO-toluene mixtures and PPO-toluene-ethyl alcohol mixtures are presented. 


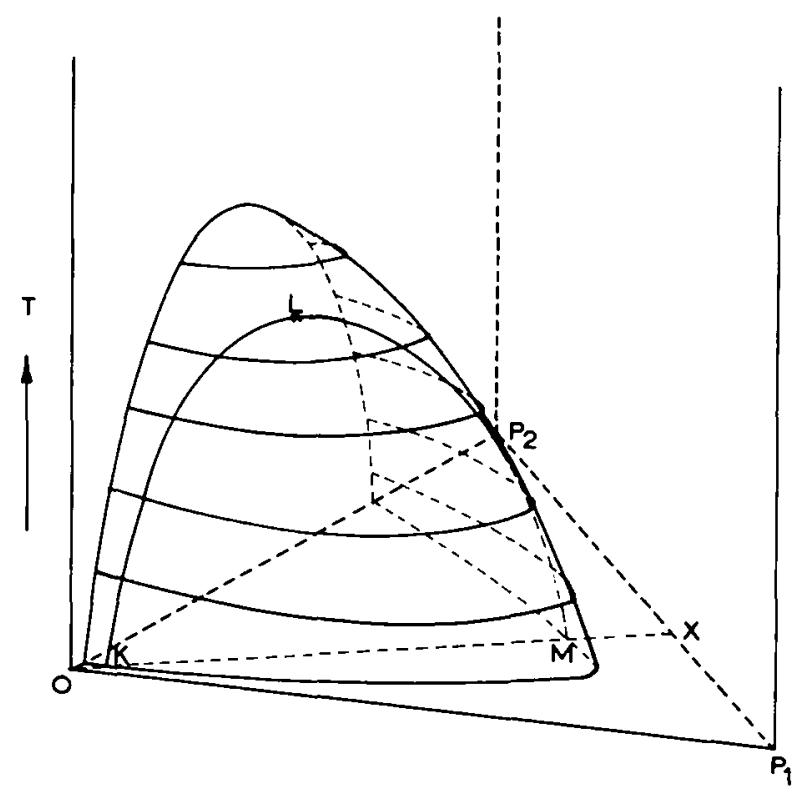

FIG. 1. Liquid ternary system: $P_{1}$, polymer with chain length $n ; P_{2}$, polymer with chain length $m(m>n) ; \mathrm{OP}_{1}$, complete miscibility; $\mathrm{OP}_{2}$, limited miscibility; $K L M$, cloud point curve.

\section{THERMODYNAMIC CONSIDERATIONS}

The free enthalpy (Gibbs free energy) of mixing $\Delta G_{m}$ of any closed system in stable equilibrium at constant pressure and temperature can be represented graphically for binary and ternary systems. In regions where $\Delta \mathrm{G}_{\mathrm{m}}$ of the homogeneous solutions is higher than that of a combination of two phases the minimum free enthalpy of the system will be given by the phase separated system; the composition of the phases being given by the tangent phase of the $\Delta G_{m}$ surface. When all sets of tangent points are projected on the base triangle, they represent the binodial which is the limit of stability of the homogeneous phase.

Another set of characteristic points of the $\Delta G_{m}$ surface are the inflection points which form the boundary of the concave part of the free energy surface. Its projection on the base triangle is called the spinodial. Phase diagrams of this type are characteristic for a nonsolvent (1)/solvent (2)/solute (3) system and have been extensively discussed by Tompa [6] .

It is clear that the composition diagram will have three regions:

1) A region where all solutions are stable $\left(\Delta G_{m}\right.$ concave upwards). This is the area outside the binodial.

2) A metastable region ( $\Delta G_{m}$ is still concave upwards, but fluctuations of a certain composition and size, may initiate phase separation). This is the area between the binodial and the spinodial. 
3) An unstable region ( $\Delta G_{m}$ is concave downwards). This is the area within the spinodial, where spontaneous phase separation always takes place by the spinodial mechanism.

A polymer solution actually represents a multicomponent system and a graphical representation of $\Delta G_{m}$ would require more than three dimensions. Several authors $[7,8]$ have shown that a three-dimensional diagram for a liquid ternary system brings out most of the essential features of the behavior of polydisperse polymer solutions. A phase diagram for a polymer solution is shown in Figure 1. At the temperatures considered, $\mathrm{OP}_{1}$ and $\mathrm{OP}_{2}$ show complete and limited miscibility, respectively. OX represents all systems containing a certain mixture of $P_{1}$ and $P_{2}$, which is the actual polymer sample, and the solvent. The plane TOX intersects the binodial surface; the resulting curve is called the cloud point curve. The spinodial must be situated below the cloud point curve, touching the cloud point curve in the critical point.

For most polymer solvent systems the critical point and the maximum of the cloud point curve are situated between 2 and $15 \%$ by weight polymer. Liquid-liquid phase separation seems to occur in most cases by nucleation and growth which is followed by coalescence of the separated liquid phase to give two bulk liquid layers in the end. If nucleation and growth is a very slow process it is possible to cool a solution below the spinodial without extensive nucleation taking place. In that case liquid-liquid phase separation will be initiated by the spinodial mechanism.

\section{EXPERIMENTAL}

The materials used were:

PPO: $\mathbf{M}_{\mathrm{n}}=25,000, \mathrm{M}_{\mathrm{w}}=39,000, \mathrm{~T}_{\mathrm{g}} \approx 220^{\circ} \mathrm{C}$; toluene; ethyl alcohol PA. Homogeneous mixtures of PPO-toluene and/or ethyl alcohol were made by weighing the appropriate amounts in small Pyrex glass tubes which were degassed, flushed with nitrogen, degassed again and sealed under vacuum at liquid nitrogen temperature. The sealed glass tubes were heated in an oil bath at $150^{\circ} \mathrm{C}$ for a minimum $24 \mathrm{hr}$ in order to get homogeneous liquid mix tures. Phase separation points were determined visually by cooling the thermostated bath by $1^{\circ} \mathrm{C}$ every $10 \mathrm{~min}$ or by $1^{\circ} \mathrm{C}$ every $48 \mathrm{hr}$. After phase separation set in, the mixtures were kept at a temperature well below the phase separation temperature for at least $24 \mathrm{hr}$ before the phase separation points were determined anew by heating the demixed solutions at the rate of $1^{\circ} \mathrm{C}$ every 10 min or of $1^{\circ}$ every $48 \mathrm{hr}$. Equilibrium concentrations could be determined for the dilute phase only. Solutions were kept at the appropriate demixing temperature for at least 2 weeks. By centrifugation at that temperature the dilute and the concentrated phase were separated. The percentage of PPO in the dilute phase could easily be determined by evaporation of the toluene. The concentrated phase could never be made completely free of the dilute phase even after prolonged periods of centrifugation, hence determination of the former phase's concentration was not feasible. 


\section{RESULTS}

\section{PPO-Toluene Mixtures}

When a homogeneous mixture of PPO in toluene is cooled two transition temperatures will be found depending upon the cooling rate. The phase separation temperatures for different mixtures observed during cooling at $1^{\circ} \mathrm{C}$ per $10 \mathrm{~min}$ are shown in Figure 2 as curve $\mathrm{A}$, which is characterized by the experimental phenomenon of instantaneous complete demixing with out formation of nuclei being detectable under the light microscope.

The transition temperatures observed during extremely slow cooling at $1^{\circ} \mathrm{C}$ each $48 \mathrm{hr}$ are shown as curve B in Figure 2. When phase separation has set in and the solution is heated again after $24 \mathrm{hr}$ of waiting, a third curve of transition temperatures will be found, curve $\mathrm{C}$ in Figure 2. This latter curve is found independently of the rate of heating.

A different way to determine curve $A$ is quenching a homogeneous mix ture of PPO-toluene to a low temperature and to determine the time which elapses before phase separation becomes visible. At higher final temperatures this incipient precipitation time suddenly increases to high values as shown in Figure 3.

A $5 \mathrm{~min}$ time limit has been adopted for the estimation of the temperatures shown in curve $A$ in Figure 2. The curves A and B are both cut off by the glass transition temperature line. Homogeneous solutions cooled to appropriate

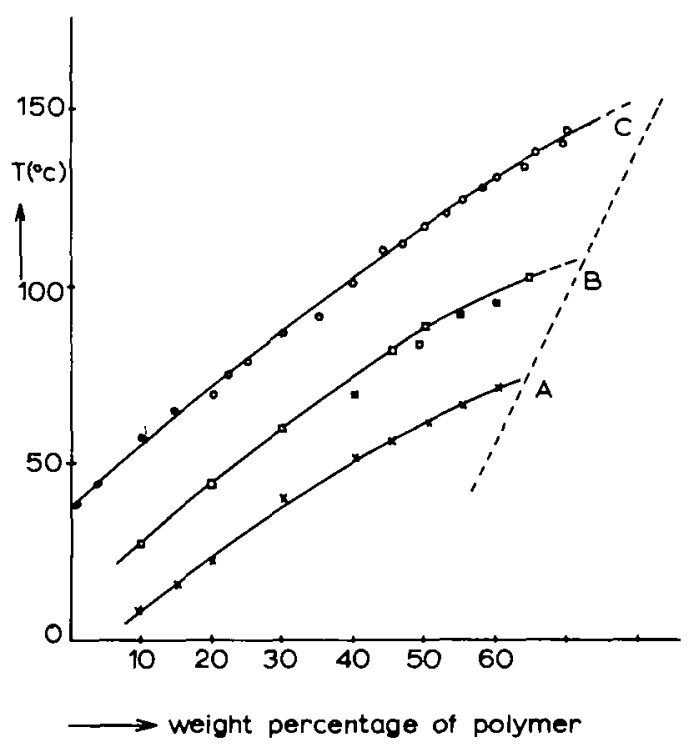

FIG. 2. Phase separation curves of PPO-toluene solutions: $(x)$ phase separation tempera tures observed during cooling at $1^{\circ} \mathrm{C}$ per $10 \mathrm{~min}$; (a) phase separation temperatures observed during cooling at $1^{\circ} \mathrm{C}$ per $48 \mathrm{hr}$; ( $(\circ)$ melting point curve; $(-)$ glass transition temperature line. 


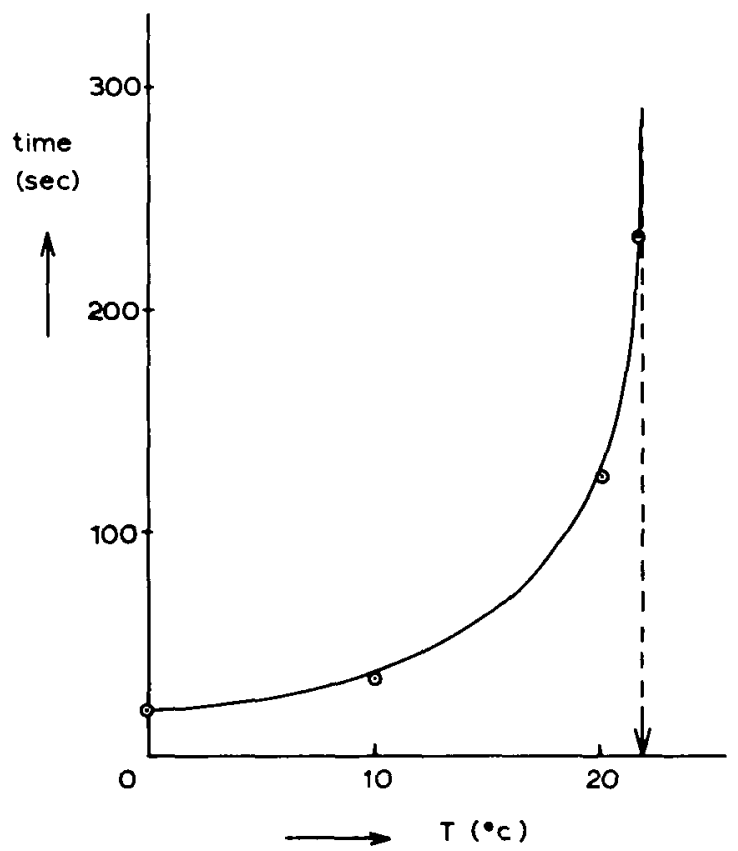

FIG. 3. Incipient precipitation time as a function of the quenching temperature of a $20 \%$ of weight PPO-toluene solution.

temperatures between curve $B$ and $C$ will remain homogeneous even after prolonged periods of at least 4 months.

Cooled at temperatures between $B$ and A liquid-liquid phase separation occurs by way of nucleation and growth (Fig. 4), nucleation occurring after induction times which approach infinity at temperatures near curve $B$ and which fall to zero when the temperature approaches curve A [9] .

The phase equilibrium curves of different PPO-toluene mixtures have been determined. Due to experimental difficulties the percentage of weight PPO could be determined in the dilute phase only (Fig. 5). These equilibrium curves finish in curve $B$ of Figure 2.

\section{PPO-Toluene-Ethyl Alcohol}

The phase separation curves for different mixtures of PPO-toluene-ethyl alcohol are shown in Figures 6, 7, 8, and 9. In Figure 6 in each solution

$$
\frac{\text { weight percent } \mathrm{PPO}}{\text { weight percent ethyl alcohol }}=1
$$

If the total amount of PPO + ethyl alcohol exceeds $40 \%$ of the total weight, liquid-liquid phase separation only occurs, independent of the cooling rate. The final situation was always characterized by two bulk liquid fractions, a 


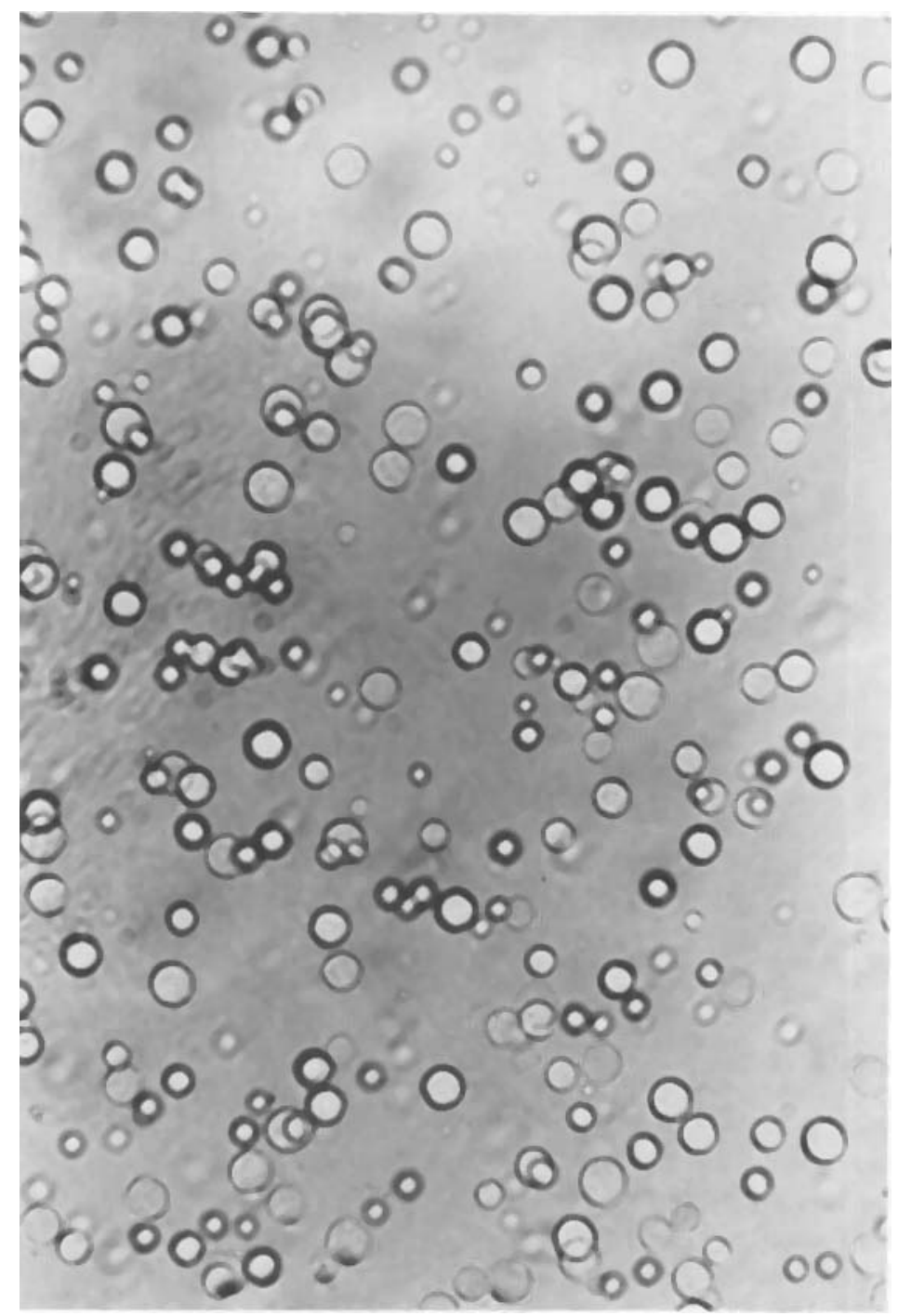

FIG. 4. Liquid-liquid phase separation by nucleation and growth of a $30 \%$ of weight PPO-toluene solution at $\mathrm{T}=48^{\circ} \mathrm{C}$.

concentrated and a dilute one. If the solutions contained less than $40 \%$ of weight PPO + ethyl alcohol, three curves could be observed in the same way as with PPO-toluene mixtures.

In Figure 7

$$
\frac{\text { weight percent toluene }}{\text { weight percent ethyl alcohol }}=
$$




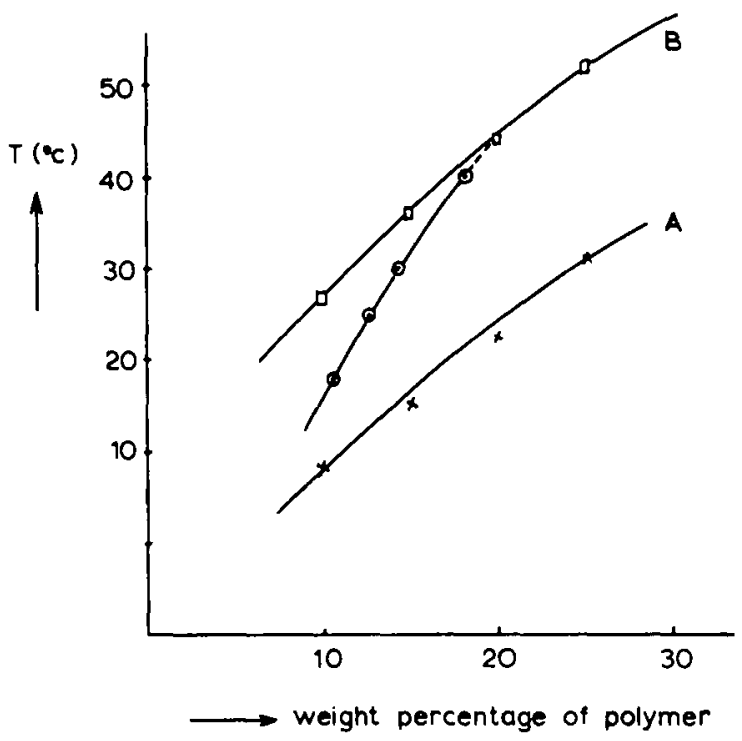

FIG. 5. Phase equilibrium of the dilute phase of demixed $20 \%$ of weight PPO-toluene solution: $(0)$ equilibrium concentration of dilute phase; ( 0 ) phase separation points observed during cooling at $1^{\circ} \mathrm{C}$ each $48 \mathrm{hr}$; $(X)$ phase separation points observed during cooling at $1^{\circ} \mathrm{C}$ per $10 \mathrm{~min}$.

Only liquid-liquid phase separation was observed leading to two bulk liquid fractions, phase separation points being independent of the speed of cooling or heating.

In Figure 8

$$
\frac{\text { weight percent toluene }}{\text { weight percent PPO }}=7 / 3
$$

and in Figure 9

$$
\text { weight percent toluene }=50 \% \text { constant }
$$

Both figures show the same features as Figure 6. In regions where the concentration of ethyl alcohol is low three curves could be determined. In the other regions liquid-liquid phase separation occurred only.

\section{DISCUSSION OF RESULTS}

In the literature many polymer-liquid systems have been discussed. In all those systems it was quite clear whether the phase separation curve was a melting point curve or a liquid-liquid phase separation curve. PPO belongs to the 


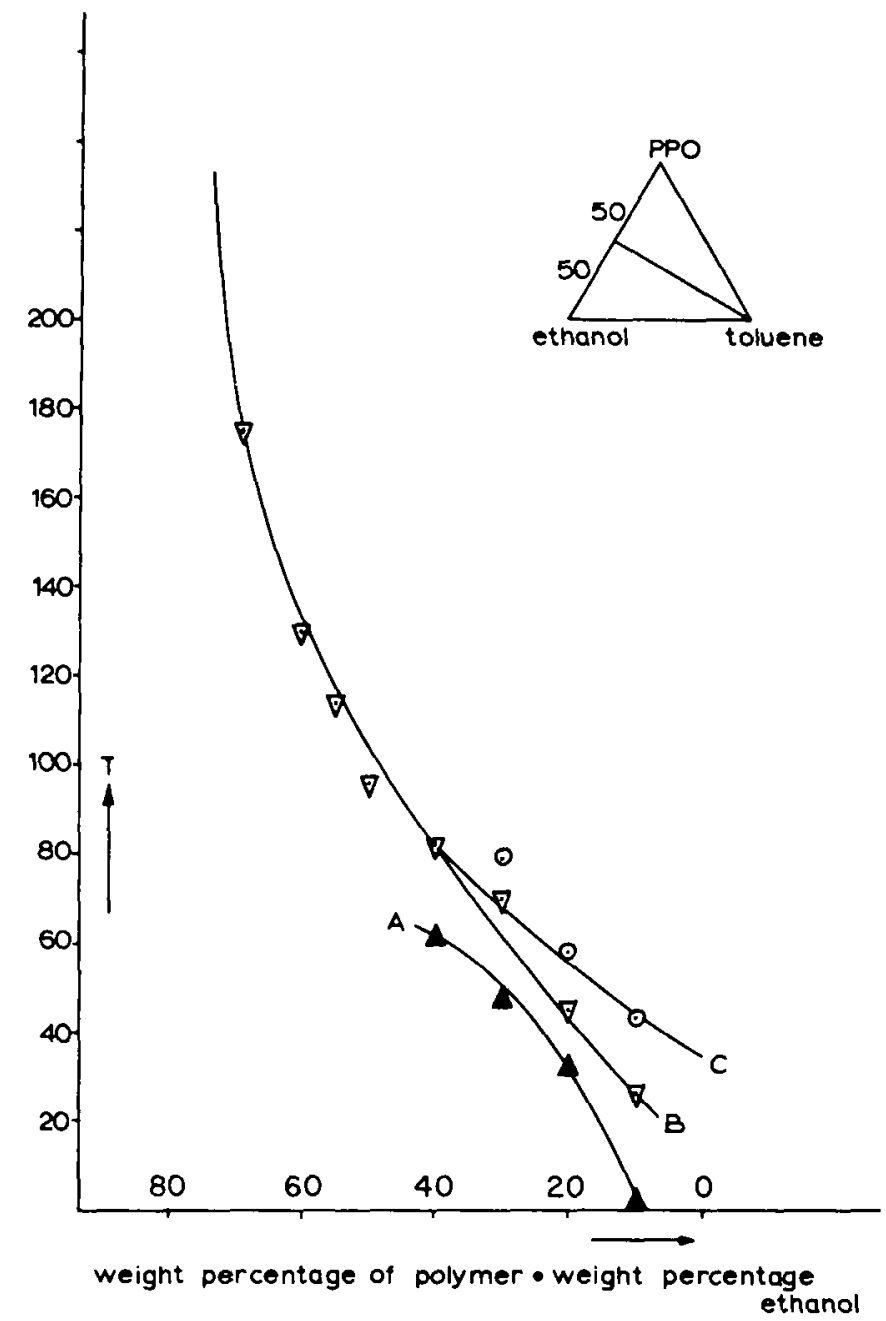

FIG. 6. Phase separation curves of PPO-toluene-ethanol solutions: ( $\nabla)$ cloud point curve; (๑) melting point curve; (^) spinodial.

class of slowly crystallizing polymers [10]. It cannot be crystallized by thermal treatment but may show crystallization when brought into contact with certain organic liquids [11]. Even then crystallization occurs very slowly.

When the system PPO-toluene is considered, the problem is how to interpretate the curves $\mathrm{A}, \mathrm{B}$, and $\mathrm{C}$.

We believe that curve B represents the cloud point curve for the following reasons.

1) The end of the different liquid-liquid phase separation curves of the system PPO-toluene-ethyl alcohol in the PPO-toluene plane is always situated on curve B.

2) When homogeneous mixtures of PPO-toluene are cooled to the appropriate temperature between curves $A$ and $B$ phase separation occurs by 


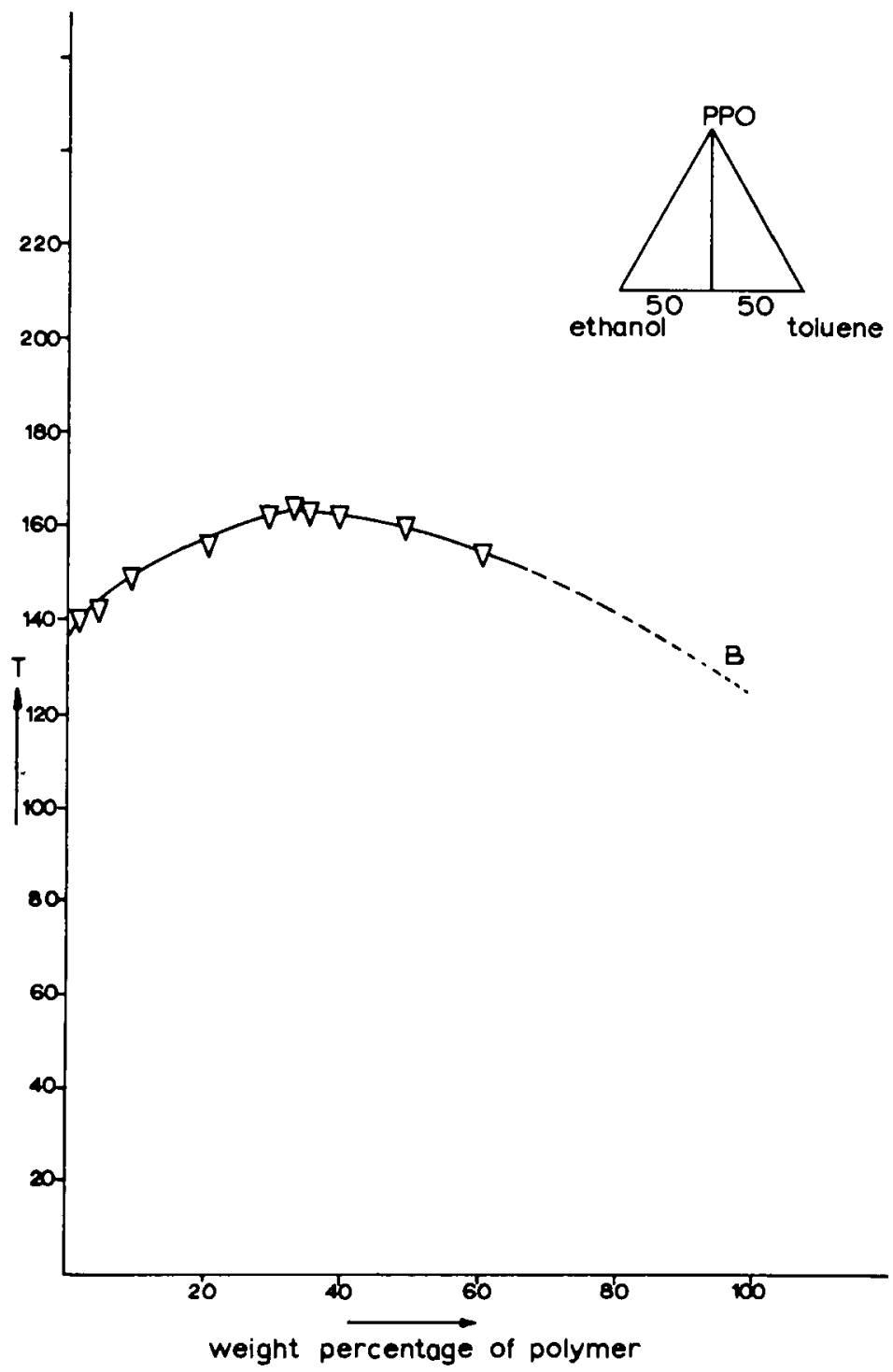

FIG. 7. Cloud point curve of PPO-toluene-ethanol solutions. In each solution the wt $\%$ ethanol $=\%$ toluene.

nucleation and growth. The spherical areas of the concentrated phase (Fig. 4) do not show any properties of spherulites (they do not show the Maltese cross under polarized light, e.o.). Only after prolonged waiting does it lighten up a bit, indicating that crystallization of the concentrated areas eventually takes place after liquid-liquid phase separation has begun.

3) The phase equilibrium curve of the dilute phase ends in curve B (Fig. 5) which is characteristic for liquid-liquid phase separation [7]. 


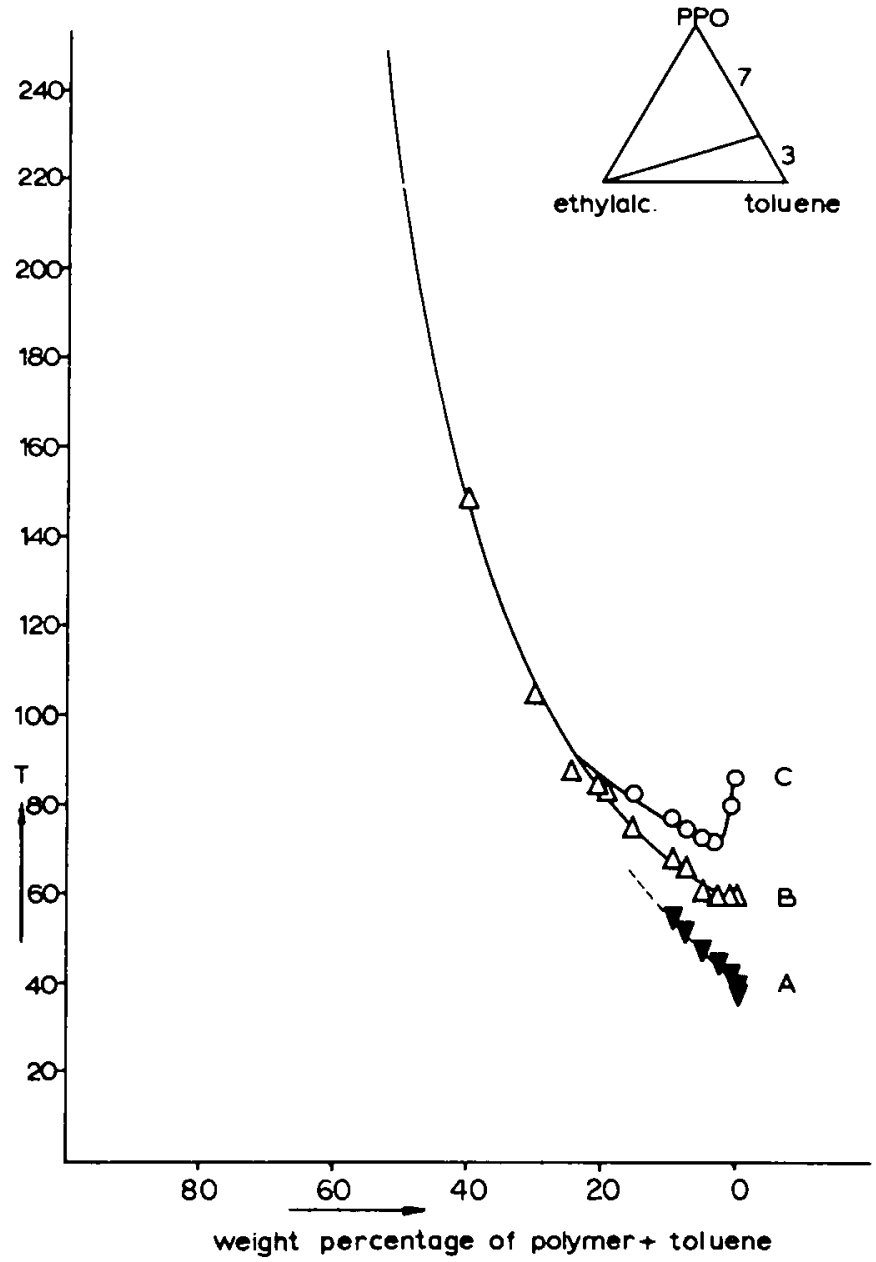

FIG. 8. Phase separation curves of PPO-toluene-ethanol solutions. In each solution

$$
\frac{\text { wt } \% \text { toluene }}{\text { wt } \% \text { PPO }}=\frac{7}{3}
$$

$(\Delta)$ cloud point curve; $(0)$ melting point curve; $(\boldsymbol{\nabla})$ spinodial.

4) Homogeneous solutions cooled rapidly at temperatures below curve $A$ demix instantaneously but will become homogeneous again at temperatures corresponding to curve $B$ when heated immediately after completion of phase separation.

Curve A represents the spinodal because

1) in homogeneous solutions of PPO in toluene rapidly cooled to temperatures on or below curve A nucleation has never been detected but structures well known for spinodial decomposition have been observed [4].

2) homogeneous mixtures of PPO in toluene demix instantaneously when cooled to any temperature below curve $A$ (except when the quenching temperature is below the $T_{g}$ of the mixture). 


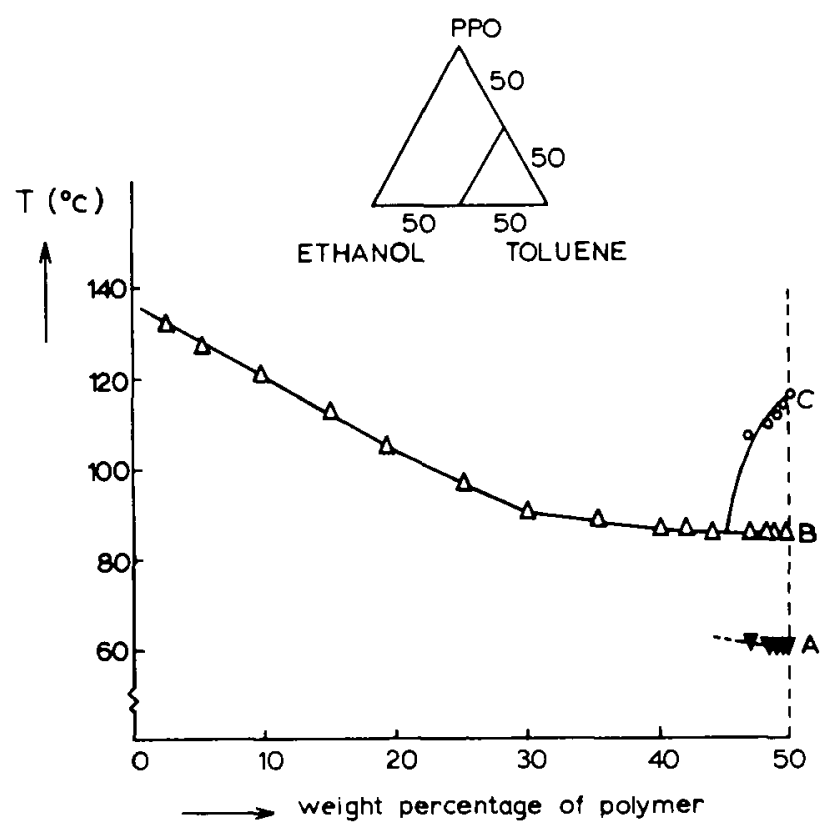

FIG. 9. Phase separation curves of PPO-toluene-ethanol solutions. In each solution the wt $\%$ toluene $=50:(\Delta)$ cloud point curve; $(0)$ melting point curve; $(\nabla)$ spinodial.

Curve $\mathrm{C}$ represents the melting point curve since

1) the concentrated areas formed during nucleation and growth lighten up a bit under polarized light when kept at the demixing temperature, indicating that crystallization is gradually taking place;

2) if these concentrated areas are kept at the demixing temperature for at least $24 \mathrm{hr}$ the concentrated phase can be separated from the dilute phase. After careful drying the residue which is pure PPO, does show some crystallinity;

3) if demixed solutions are kept at the demixing temperature for various times, the transition temperatures determined when the solutions are heated again, are always situated on curve $\mathrm{C}$, never at temperatures between $\mathrm{B}$ and $\mathrm{C}$.

The most remarkable feature of the system PPO-toluene is the cloud point curve B in Figure 2, which does not show a maximum up to $75 \%$ of weight PPO, which is quite different from cloud point curves known from literature. A possible explanation for this phenomenon is an unusual dependence of the interaction between polymer segments and solvent molecules on concentration and temperature [13]. Taking curves $A$ and $B$ into account, we propose that Figure 10 depicts the phase diagram of the liquid system PPO-toluene.

The phase separation curves of the system PPO-toluene-ethyl alcohol represent the cloud point curves of this system. Only in regions close to the PPO-toluene plane splitting up into three curves takes place. Curve $\mathrm{C}$ is again the melting point curve, curve $B$ is the cloud point, and curve $A$ is the spinodial.

Cross sections of the diagram of this system at different temperatures are given in Figure 11. From Figure 11 it can be read that depending upon the 


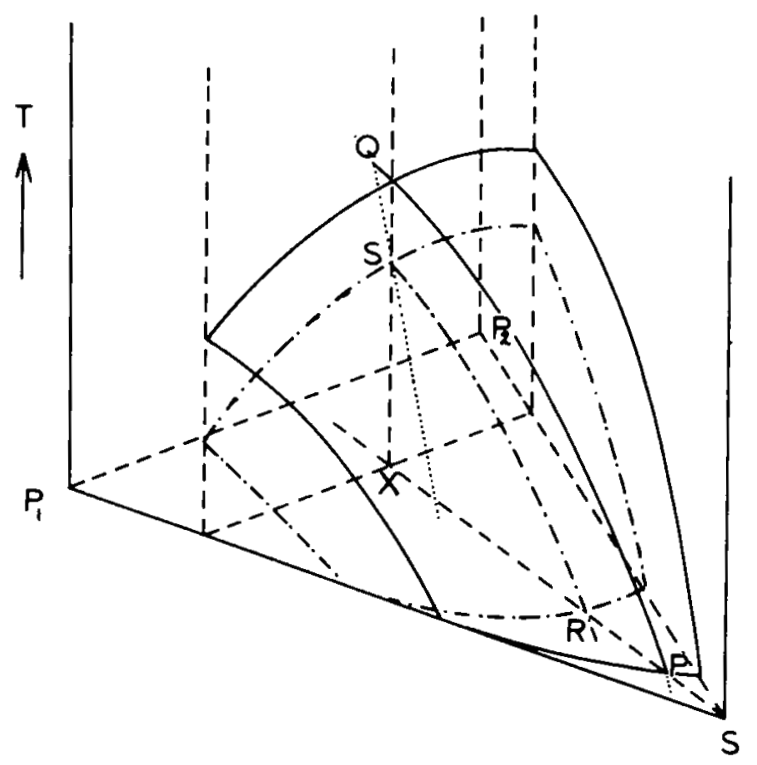

FIG. 10. Ternary liquid diagram for PPO-toluene: $P_{1}$, polymer chain length $n ; P_{2}$, polymer chain length $m(m>n) ; S$, solvent; $P Q$, cloud point curve; RS, spinodial curve.

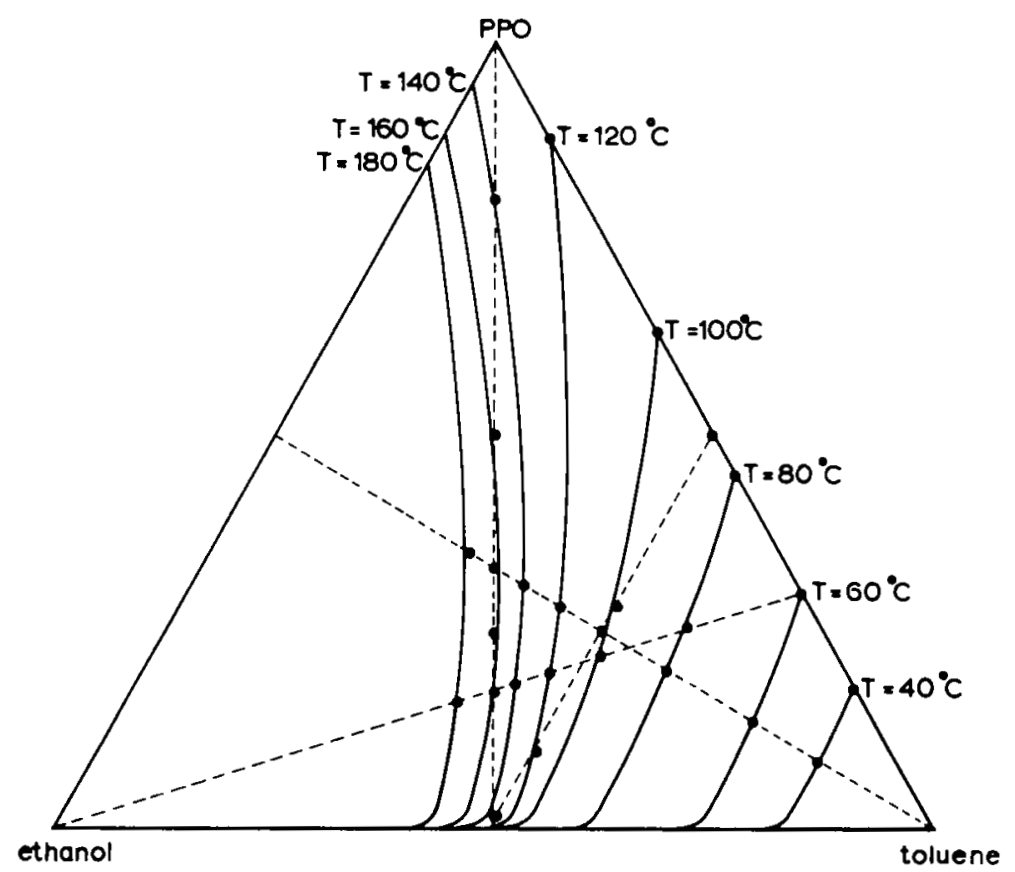

FIG. 11. Cross sections at different temperatures of the phase diagram of the system PPO-toluene-ethanol. 
temperature a considerable amount of the extremely poor solvent ethyl alcohol can be added to mixtures of PPO-toluene without causing a phase separation. If phase separation occurs the final situation is characterized by two bulk liquid fractions except for regions close to the PPO-toluene plane. Nucleation and growth are not retarded any more and the spinodial cannot be measured.

Even small amounts of ethyl alcohol causes a considerable depression of the melting points until below the liquid-liquid separation line. Only in solutions containing small amounts of ethyl alcohol compared to toluene, is it possible to determine experimentally the three curves mentioned above.

For temperatures above $130^{\circ} \mathrm{C}$ the phase diagram in Figure 11 is the same as discussed by Tompa [6] for the general case polymer/solvent/nonsolvent. Below $130^{\circ} \mathrm{C}$ the diagram differs from this system and is also different when compared with the diagram of the general system polymer/nonsolvent/nonsolvent as discussed by Tompa and Scott [12] .

\section{CONCLUSIONS}

From these results it can be inferred that in the system PPO-toluene the amorphous separation range is situated close to the melting point curve of the crystalline polymer. In their paper on classification of polymer solvent systems Papkov and Yefimova [14] give a general description of such a system that is in good accordance with the system PPO-toluene. If a homogeneous solution of such a system is cooled to a temperature below its melting point phase separation will not occur because the rigid chains of the polymer prevent the precise molecular packing necessary for crystallization. Upon further cooling the metastable range with respect to liquid-liquid phase separation is entered into where a simple piling up of molecules is sufficient to reach the required critical concentration and size. As a result liquid-liquid phase separation is initiated by nucleation and growth and two amorphous phases are formed. One phase (the most concentrated one) corresponds now to very high supersaturation with respect to crystallization and creates conditions for spontaneous nucleation of the crystalline phase.

The spinodial can only be detected if during cooling nucleation occurs extremely slowly. In PPO-toluene solutions this is actually the case and spinodial decomposition patterns are observed. A gel is formed which is builtup of interconnected areas of the concentrated and the dilute phase. The concentrated areas correspond again to high supersaturation with respect to crystallization and crystallizes. As a result the gel changes into a rigid mass, in this way preserving the spinodial pattern. Further microscopic and light scattering experiments are in good agreement with the experiments and conclusions described in this article [13].

\section{REFERENCES}

[1] M. Volmer and A. Weber, Z. Phys. Chem., 119, 277 (1925).

[2] J. W. Cahn, Trans. Met. Soc. AIME, 242, 166 (1968). 
[3] J. J. van Aartsen and C. A. Smolders, Europ. Polym. J. 6, 1105 (1970).

[4] C. A. Smolders, J. J. van Aartsen, and A. Steenbergen, Kolloid-Z. Z. Polym., 243, 14 (1971).

[5] J. J. van Aartsen, Europ. Polym. J., 6, 919 (1970).

[6] H. Tompa, Polymer Solutions, Buttworths, London, 1956.

[7] R. Koningsveld, Advan. Coll. Interface Sci, 2, 151 (1968).

[8] G. Rehage, D. Moller, and O. Emst, Makromol. Chem, 88, 232 (1965).

[9] P. T. van Emmerik and C. A. Smolders, to be published.

[10] J. Boon and E. P. Magre, IUPAC Inter. Symp., vol. II, Leiden, 834 (1970).

[11] F. E. Karasz and J. M. O'Reilly, J. Polym. Sci, B3, 561 (1956).

[12] R. L. Scott, J. Chem. Phys., 17, 3, 268 (1949).

[13] P. T. van Emmerik and C. A. Smolders, J. Polym Sci., C., 39, to be published.

[14] S. P. Papkov and S. G. Yefimova, Faserforsch Textil. tech. Sowj. Beitrage, 4, 118 (1967). 